\title{
Las destrezas lingüísticas a través del análisis publicitario en clase de español para fines profesionales
}

Ana-Isabel Ribera Ruiz de Vergara

\section{(2) OpenEdition \\ Journals}

Edición electrónica

URL: http://journals.openedition.org/esp/609

DOI: 10.4000/esp.609

ISSN: 2532-0319

Editor

Centre d'Information sur l'Éducation Bilingue et Plurilingue

\section{Edición impresa}

Fecha de publicación: 1 diciembre 2015

Paginación: 35-42

ISSN: 1127-266X

\section{Referencia electrónica}

Ana-Isabel Ribera Ruiz de Vergara, « Las destrezas lingüísticas a través del análisis publicitario en clase de español para fines profesionales », Éducation et sociétés plurilingues [En línea], 39 | 2015, Puesto en línea el 01 octubre 2016, consultado el 01 mayo 2019. URL : http:// journals.openedition.org/esp/609; DOI : 10.4000/esp.609 


\section{LAS DESTREZAS LINGÜÍSTICAS A TRAVÉS DEL ANÁLISIS PUBLICITARIO EN CLASE DE ESPAÑOL PARA FINES PROFESIONALES}

\section{Ana-Isabel Ribera RUIZ DE VERGARA}

Uno degli obiettivi degli studenti che vengono all'università per seguire un corso di laurea in lingue straniere applicate, in questo caso in spagnolo, è quello di avere una padronanza sicura della lingua e di usarla come mezzo di comunicazione nel mondo del lavoro. Il problema sorge quando l'insegnante di lingua si trova di fronte ad una classe in cui gli studenti hanno livelli eterogenei e quindi gli si pone il compito di rendere la classe più omogenea. In quest'articolo proporremo un'attività nell'ambito dell'analisi pubblicitaria che faciliterà agli studenti di lingue straniere applicate la pratica della competenza linguistica comunicativa a partire dal terzo trimestre di studio della lingua. Questo tipo d'attività è concepito per gli studenti la cui specialità ha un legame con il marketing, la pubblicità, la stampa e la comunicazione.

Parole chiavi: Lingue straniere applicate, mondo del lavoro, classe eterogenea, analisi pubblicitaria

Un des objectifs des étudiants qui viennent à l'université pour suivre un cursus en Langues Étrangères Appliquées (LEA), en l'occurrence l'espagnol, est de réussir à maîtriser la langue et de s'en servir comme moyen d'expression dans le monde du travail. Un problème survient lorsque le professeur de langue se trouve face à une classe dont les étudiants ont un niveau très hétérogène et qu'il doit parvenir à harmoniser les différents niveaux. Dans cet article on proposera une activité en lien avec l'analyse publicitaire qui facilitera aux étudiants de la filière LEA la pratique de la compétence linguistique communicative à partir du troisième semestre d'études de la langue. Ce type d'activité est conçu pour les étudiants dont la spécialité a un rapport avec le marketing, la publicité, la presse et la communication.

Mots clés: Langues étrangères appliquées, monde du travail, classe hétérogène, analyse publicitaire

One of the aims of students following an LEA (Applied Foreign Languages) curriculum at University is to master the language (Spanish here) to be able to use it professionally. A problem crops up when the teacher finds him/herself in front of a class where several levels obtain. In this article an activity is proposed connected to the analysis of publicity ads. Made for students in Marketing, Advertising, the press and communications, the program helps LEA students sharpen their communicative skills as of the third semester.

Key words: Applied foreign languages, working world, multi-level classes, publicity analysis

INTRODUCGIÓN
C Yomo señalan autores y profesores dedicados a la enseñanzaaprendizaje del Español con Fines Profesionales (EFP), Aguirre Beltrán (2012) por ejemplo, el objetivo de la Didáctica del EFP es preparar a los estudiantes para que integren el mundo profesional 
Las destrezas lingüísticas a través del análisis publicitario en clase de español para fines profesionales

A.-I. Ribera Ruiz de Vergara

DIDÁCTICA DE EFP EN LOS DEPARTAMENTOS UNIVERSITARIOS DE LEA

CARACTERÁSTICAS CONTEXTUALES GENERALES DEL ESTUDIANTADO EN LOS PRIMEROS SEMESTRES DE EFP EN LOS DEPARTAMENTOS UNIVERSITARIOS DE LEA sabiendo utilizar la lengua española.

Y, según dejan entender los estudiantes de la rama universitaria de Lenguas Extranjeras Aplicadas (LEA), su objetivo es servirse del español para ejercer en un país hispanófono, pudiendo comunicarse tanto en la lengua escrita como en la lengua oral, utilizando, con desenvoltura, la lengua general y la lengua de especialidad.

Queda claro, pues, que el objetivo de la Didáctica del EFP y el objetivo que dicen querer alcanzar muchos estudiantes de LEA coincide.

En este apartado vamos a citar, primero, algunas características contextuales generales de los estudiantes en primer año de departamentos universitarios de LEA que hacen que la didáctica de la lengua extranjera, en nuestro caso el Español, no se dé siempre en las circunstancias más idóneas. Después, veremos qué piensan realmente los estudiantes de EFP en los departamentos de LEA con respecto a la enseñanzaaprendizaje del Español y cuáles son sus expectativas.

Para reflejar de manera clara el contexto en el que se realiza la didáctica de la lengua, en nuestro caso EFP, en los departamentos universitarios de LEA, en el cuadro que exponemos a continuación hemos querido comparar los factores que determinan las características contextuales de los departamentos universitarios de LEA con otros centros, por ejemplo, las Escuelas Superiores de Comercio (ESC), en donde, a menudo, se lleva a cabo una didáctica idéntica o parecida del EFP.

\begin{tabular}{|c|c|}
\hline Kstudiantes de ESC & Fstudiantes de LEA \\
\hline BAC +2 años de preparación de acceso & BAC + acceso directo a $1^{\sigma}$ año \\
\hline + una «oposición» (concours) de acceso & No hay uoposicióm» de acceso \\
\hline $1^{\text {er }}$ año $\mathrm{ESC} \equiv \mathrm{BAC}+3$ & $\mathbf{I}^{\text {ex }}$ año $\mathbf{L E A} \equiv \mathrm{BAC}+1$ \\
\hline Nivel: $\mathrm{B} 2 \pm$ & Nivel: $\mathbf{A} 1-\mathbf{A} 2 \pm$ \\
\hline
\end{tabular}

Figura 1

Como se desprende de la figura 1, los estudiantes de LEA llegan directamente del BAC a la universidad sin haber pasado una prueba de acceso que se prepara en dos años y que, en cambio, sí deben superar los estudiantes que quieren acceder a otros centros de enseñanza superior, como Escuelas Superiores de Comercio, en donde se realiza un tipo de enseñanza similar a la de LEA. Los estudiantes de LEA cuentan, por lo tanto, en su haber, con menos horas de enseñanza-aprendizaje de español. A esto hay que añadir la heterogeneidad en el nivel, al menos, durante los dos primeros semestres de aprendizaje de la lengua en LEA. 
Las destrezas lingüísticas a través del análisis publicitario en clase de español para fines profesionales

A.-I. Ribera Ruiz de Vergara
Del recuadro que exponemos a continuación, en la figura 2, podemos destacar que a los estudiantes de los departamentos de LEA les resulta más fácil el aprendizaje de la lengua especializada (el aprendizaje del léxico relacionado con su especialidad, por ejemplo) que la lengua general. Consideran, en su gran mayoría, que es muy importante el aprendizaje de la lengua general. Únicamente el dos por ciento de los entrevistados opina que lo más importante para ellos es el aprendizaje de la lengua especializada, dejando de lado la lengua general. Admiten, en su mayoría, que su nivel en lengua española no es elevado y que no le dedican mucho tiempo al estudio personal de la lengua.

\begin{tabular}{|c|c|}
\hline $\begin{array}{l}\text { Preguntas formuladas a } 100 \text { estudiantes, al término del primer año de estudios } \\
\text { en IEA }\end{array}$ & Respuestas \\
\hline ¿Cuál es, en tu opinión, tu nivel en español a tu llegada a la universidad? & En general opinan que no es muy alto \\
\hline ¿Le dedicas tiempo personal al estudio del español? ¿Cuánto tiempo? & $\begin{array}{l}\text { La mayoría admite dedicarle poco tiempo, o } \\
\text { incluso nada, al estudio del español }\end{array}$ \\
\hline ¿Cuáles son tus aspiraciones de trabajo para el futuro? & $\begin{array}{l}\text { Por orden de preferencia: } \\
\text { 1. La mayoría no sabe aún con exactitud } \\
\text { qué quiere hacer } \\
\text { 2. Turismo } \\
\text { 3. Trabajar en una empresa. } \\
\text { 4. Trabajar en el mundo de la comunicación } \\
\text { 5. Buscar, primero, un trabajo en el } \\
\text { extranjero }\end{array}$ \\
\hline ¿Qué lugar ocupa el español en tus aspiraciones futuras de trabajo? & $\begin{array}{l}\text { Consideran, en su mayoría, que el inglés } \\
\text { ocupa un lugar más importante que el } \\
\text { español en sus aspiraciones futuras de } \\
\text { trabajo }\end{array}$ \\
\hline $\begin{array}{l}\text { ¿Qué te resulta más fácil con respecto al aprendizaje del español: la lengua } \\
\text { relacionada con tu especialidad o la lengua general? }\end{array}$ & La lengua relacionada con su especialidad \\
\hline $\begin{array}{l}\text { ¿Qué te parece más importante con respecto al aprendizaje del espan̄ol: } \\
\text {-aprender únicamente la lengua relacionada con tu especialidad, } \\
\text {-aprender la lengua general, } \\
\text {-las dos anteriores? }\end{array}$ & $\begin{array}{l}75 \% \text { aprender la lengua general, } \\
23 \% \text { aprender las dos: la lengua general y la } \\
\text { lengua de especialidad, } \\
2 \% \text { únicamente la lengua de especialidad }\end{array}$ \\
\hline $\begin{array}{l}\text { ¿Estudias la lengua española para poder ejercer tu trabajo en un pais } \\
\text { hispanófono? }\end{array}$ & Una gran mayoria contesta que si \\
\hline
\end{tabular}

\section{OPINIÓN Y}

EXPECTATIVAS DE

LOS ESTUDIANTES

DE EFP EN LOS

DEPARTAMENTOS

UNIVERSITARIOS DE

LEA
Figura 2

Del recuadro de la figura 2, se destaca también que, si la ocasión se les presentara a nuestros estudiantes de LEA, les gustaría trabajar en un país hispanófono pudiendo utilizar adecuadamente las competencias lingüisticas. A partir de estas expectativas, dos preguntas se nos plantean:

Primera pregunta: ¿Cómo ayudar al estudiantado de los departamentos universitarios de LEA, a alcanzar el que dice ser su objetivo y facilitarle la práctica y, por consiguiente, el aprendizaje de las destrezas lin- 
Las destrezas lingüísticas a través del análisis publicitario en clase de español para fines profesionales

A.-I. Ribera Ruiz de Vergara

LENGUA GENERAL Y LENGUA APLICADA EN EFP A TRAVÉS DEL ANÁLISIS PUBLICITARIO

¿POR QUÉ PROPONER TAREAS RELACIONADAS CON EL ANÁLISIS PUBLICITARIO EN EFP? gǘsticas de la competencia comunicativa, o lo que es lo mismo, de la lengua general?

Segunda pregunta: ¿Cómo aplicar la lengua general al contexto profesional en el que potencialmente se verá implicado el estudiante?

En el apartado siguiente, vamos a presentar una serie de preguntas que sirven de base tanto para practicar la lengua general como la lengua aplicada.

Consideramos que el profesor encargado de hacer que el estudiante practique las destrezas lingüísticas del Español para Fines Profesionales en LEA realiza una doble tarea: la de enseñar la lengua general y, al mismo tiempo, la de enseñar la lengua aplicada al contexto profesional que podrá concernir, en un futuro, a los estudiantes. En el caso de LEA, la enseñanza de la lengua suele ir asociada a la economía y al mundo de la empresa, a la mercadotecnia, a la publicidad y a la comunicación.

Las preguntas que hemos creado, y que vamos a presentar a continuación, se refieren al análisis publicitario. Esta serie de preguntas puede, además, servir de fuente de ideas a profesores de EFP en LEA a la hora de crear sus propias experiencias prácticas de aplicación de la lengua general a la publicidad.

Porque, por un lado, mediante la práctica de la lengua a través del análisis publicitario, el estudiante afianza los conocimientos de la lengua general y, al mismo tiempo, se familiariza y profundiza los conocimientos relacionados con la lengua aplicada al dominio profesional que le interesa (recordemos que, en el caso que nos ocupa, se trata de la especialidad del marketing y la mercadotecnia, la publicidad, la prensa y la comunicación). Y porque, por otro lado, este tipo de actividad va a tener en cuenta el aprendizaje de las habilidades lingüísticas de la competencia comunicativa a partir del Marco Común Europeo de Referencia para las lenguas (MCERL). En efecto, todas las actividades de la lengua, citadas por el MCERL y relacionadas con la competencia lingüística comunicativa, se pueden realizar a través de las tareas relacionadas con el análisis publicitario, a saber, la comprensión, la expresión, la interacción o la mediación (Centro Virtual Cevantes: 212). 
Las destrezas lingüísticas a través del análisis publicitario en clase de español para fines profesionales

A.-I. Ribera Ruiz de Vergara
Las actividades de la lengua relacionadas con la competencia lingüística comunicativa se hallan bien representadas en el esquema de Evelyne Rosen (2010: 42), que aparece también en el Cadre Européen Commun de Réference francés (CECR), y que nosotros hemos adaptado y exponemos a continuación:

\section{Adaptación del esquema de Rosen}

$\begin{aligned} & \text { Operaciones } \\ & \text { mentales }\end{aligned}$
$\begin{aligned} & \text { Actividades } \\ & \text { de decodificación/ } \\ & \text { codificación del mensaje }\end{aligned}$

Figura 3

PREGUNTAS PARA

REALIZAR TAREAS

RELAGIONADAS CON EL ANÁLISIS

PUBLICITARIO EN EL AULA DE EFP
He aquí una serie de preguntas que sirven para analizar la publicidad escrita (prensa escrita, carteles, vallas publicitarias, cierto tipo de publicidad en Internet), la publicidad radiofónica y la publicidad audiovisual.

Esta actividad se divide en siete apartados:

I. Análisis del texto

- Vocabulario.

- Comprensión.

- Léxico (estudio del léxico propio de la publicidad: sencillo, de fácil comprensión, bien pronunciado, en negrita, en cursiva, en mayúsculas, en minúsculas, etc.)

- Estructuras lingüísticas.

- Sintaxis simple (frases simples, cortas, sencillas: de fácil comprensión.)

- Verbos (pocos; tiempos: presente, futuro próximo, futuro -acontecimiento que se producirá con seguridad-, imperativo -interpela directamente al lector, oyente, telespectador-; personas: $1^{\mathrm{a}}$ y $2^{\mathrm{a}}$ personas; verbo impersonal haber -hay-; etc.)

- Pronombres personales $\left(2^{\mathrm{a}}\right.$ persona, $1^{\mathrm{a}}$ persona, tuteo, tratamiento de usted, etc.) 
Las destrezas lingüísticas a través del análisis publicitario en clase de español para fines profesionales

A.-I. Ribera Ruiz de Vergara
- Pronombres y adjetivos demostrativos (de cercanía.)

- Numerales (cantidades, porcentajes, etc.)

- Pronombres y adjetivos de cantidad (todo, mucho, poco, etc.)

- Pronombres exclamativos e interrogativos (interpelan directamente al lector, oyente, telespectador, etc.)

- Signos de exclamación y de interrogación (tener en cuenta la entonación en la publicidad audio.)

- Direcciones. (Ayuda a localizar el producto.)

- ¿Valora lo tradicional y/o lo moderno?

- ¿El texto se adecúa o no al mensaje que se quiere transmitir? ¿Por qué?

II. Análisis de la imagen

- ¿Quién aparece en la imagen? (personas conocidas, anónimas, etc.)

- ¿Qué aparece en la imagen? (animales, plantas, objetos, etc.)

- ¿Cómo aparecen estas personas y/o animales y/u objetos en la imagen? (descripción completa, sin olvidar los colores, en blanco y negro, etc.)

- ¿Dónde? (descripción del lugar representado en la imagen.)

- ¿Por qué? (argumentar por qué las personas y/o animales y/u objetos de la imagen se adaptan al texto y al mensaje que se desea transmitir.)

- ¿Para qué? (finalidad de la imagen.)

(A veces, ¿por qué? y ¿para qué? se pueden explicar de manera conjunta.)

- ¿Cuándo? (explicar si la imagen se adapta o no al hoy en día.)

- ¿Valora lo tradicional y/o lo moderno?

- ¿Qué relación hay entre la imagen y el texto?

- ¿La imagen se adecúa o no al mensaje que se quiere transmitir? ¿Por qué?

III. Análisis de la voz/voces, música, otros ruidos

- Voz (masculina, femenina, joven, evocadora, alegre, triste, incitadora/provocadora, grave, aguda, natural, origen - pronunciación, entonación-, etc.)

- Música (ritmo, origen, frecuencia, antes y/o después y/o durante el texto leído o interpretado, etc.)

- Otros ruidos (corazón, motor de un coche, risas, hojas, hielo, etc.)

- ¿Cómo se adaptan la voz, la música y los otros ruidos al texto leído y/o interpretado? ¿Qué relación hay entre la voz, la música, los otros ruidos y el texto?

- ¿Para qué? (finalidad de la voz, la música y los otros ruidos.)

- ¿La voz, la música y los otros ruidos se adecúan o no al mensaje que se quiere transmitir? ¿Por qué? 


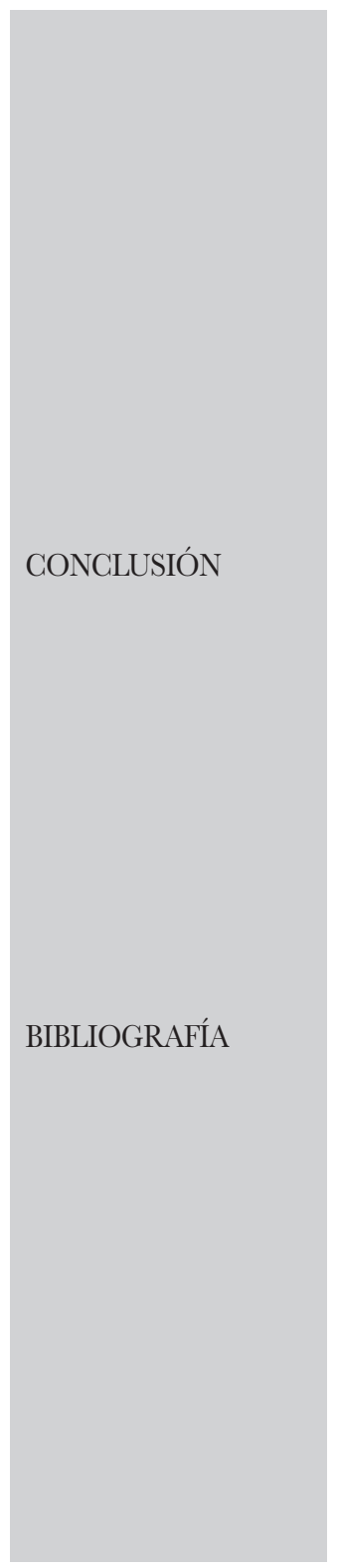

IV. ¿A qué tipo de persona se dirige esta publicidad?

- Por ejemplo, dependiendo del horario, la publicidad radiofónica atrae a amas de casa y a jóvenes.

- ¿Qué principios de las teorías AIDA y Pirámide de Maslow adopta la publicidad para atraer al tipo de persona al que se dirige? (Lengua aplicada al dominio de la publicidad.)

$\mathrm{V}$. ¿A través de qué canal de comunicación?

- Para contestar a esta pregunta, hay que tener en cuenta los paradigmas de las estrategias publicitarias. (Lengua aplicada al dominio de la publicidad.

VI. ¿Qué estrategia publicitaria adopta esta publicidad?

VII. Opinión y debate.

- En tu opinión, ¿esta publicidad consigue su objetivo? ¿Por qué?

Para concluir, queremos señalar que en las actividades relacionadas con el análisis publicitario no se hace una diferencia tajante entre la lengua general y la lengua de especialidad. En este tipo de actividades se pone en práctica la lengua en uso, que es como define Pierre LERAT a la lengua con fines profesionales (Lerat 1995). Además de perfeccionar el aprendizaje de la lengua general y de perfeccionar su aplicación al dominio profesional del marketing y la mercadotecnia, la publicidad, la prensa y la comunicación, este tipo de actividad desarrolla el pensamiento crítico y creativo del estudiante. Pero este último punto, que no hemos tratado aquí, (el del pensamiento crítico y la creatividad a través del análisis publicitario) daría materia para ser tratado en otro artículo.

AGUIRRE BELTRÁN B. 2012. Aprendizaje y enseñanza de español con fines específicos. Madrid, SGEL.

LERAT P. 1995. Les Langues spécialisées. Paris, Presses Universitaires de France.

RIBERA RUIZ DE VERGARA A. 2014. La práctica de las destrezas lingüísticas de la competencia comunicativa a través del análisis publicitario en Español para Fines Profesionales (ponencia), El español y el mundo del trabajo, XII Encuentro Internacional GERES, Metz (Francia), 19-21 de junio de 2014.

ROSEN E. 2010. Le point sur le Cadre européen commun de référence pour les langues. Paris, CLE international. 
Las destrezas lingüísticas a través del análisis publicitario en clase de español para fines profesionales

A.-I. Ribera Ruiz de Vergara

\section{SITOGRAFÍA}

http://cvc.cervantes.es/ensenanza/biblioteca_ele/marco/cap_02.htm (sitio consultado el 24 de octubre de 2014)

http://fr.wikipedia.org/wiki/Cadre_europ\%CC3\%A9en_commun_de _r $\%$ C $3 \%$ A9f $\% \mathrm{C} 3 \% \mathrm{~A} 9 \mathrm{rence}$ _pour_les_langues (sitio consultado el 24 de octubre de 2014).

\section{NOTA}

Las figuras 1 y 2, que aparecen en este artículo, fueron expuestas por Ana Isabel Ribera Ruiz de Vergara en una ponencia sobre El español y el mundo del trabajo, en el XII Encuentro Internacional del GERES que tuvo lugar en Metz (Francia) los días 19, 20 y 21 de junio de 2014. 